\title{
In the Spotlight: Biomedical Imaging
}

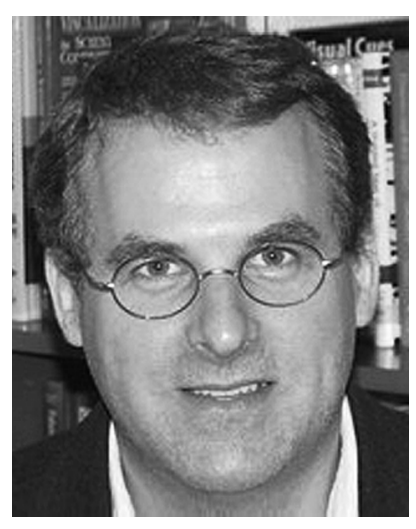

\section{INTRODUCTION}

$\mathbf{T}$ HIS year we have seen efforts geared towards developing imaging and therapeutic techniques for minimal invasiveness and improved image quality and novel software tools for image processing, analysis and storage. All of which designed to facilitate the work flow of the radiologist, better model biological systems at the cellular level, and understand complex metabolic and functional processes. Researchers in the field of biomedical imaging continue to strive to accommodate the often conflicting needs of patients, clinicians and health care costs. In addition, we have seen in emergent imaging technology a continued shift from 2-D towards 3-D plus time (4-D) modalities which require higher dimensional processing and visualization demands.

While in future spotlight articles we will focus on more immediate advances in imaging technology, it seems appropriate that this first spotlight give a more general overview of tends over a slightly broader period of time.

\section{Advances IN Biological IMAGing}

During the last decade systems biology has matured into a distinct field and imaging has been increasingly used to enable the interplay of experimental and theoretical biology. Systems biology [1] attempts to model the dynamics and structure of complete biological systems. Imaging is increasingly used to measure physical parameters such as concentration, tissue properties, and surface area and to glean temporal insight on biological function. Molecular probes can also be employed to allow for both therapeutic and diagnostic applications [2]. As the spatial resolution and acquisition frequency of imaging techniques

Manuscript received October 06, 2008. Current version published December 02, 2008.

A. F. Laine is with the Department of Biomedical Engineering, Columbia University, New York, NY 10027 USA (e-mail: laine@ columbia.edu).

Digital Object Identifier 10.1109/RBME.2008.2008221 increase, using imaging to monitor substrate and protein dynamics in real time may be more readily achieved. Such data acquired by imaging can provide the basis for mathematical modeling of protein kinetics and biochemical signaling networks [3]. Imaging can also be a suitable means to test computational models already developed as well as validating empirical insight on biological processes.

Major efforts are underway to apply systems biology methods to oncology. Increasingly sophisticated and accessible genomics, proteomics, and metabolomic high throughput experiments provide a basis for new types of oncology research. The number of published results based on gene expression microarray data alone has increased by a factor of $1700 \%$ over the last decade. These advances in experimental systems biology coupled with new analysis techniques and quantitative imaging software tools are helping to generate a more complete picture of much cancer related signaling pathways. The dream is to discover preemptive techniques for specific patients at risk.

Molecular imaging enables the determination of both temporal and spatial distributions of biological processes throughout an intact living subject. With this approach, it is possible to obtain more meaningful results than achieved by comparable in vitro methods. With recent advances in molecular imaging techniques, properly tagged molecules can be visualized leading to insights on cell function, membrane binding sites, and the effectiveness of particular therapies. In parallel, taking a look on a higher scale on how the human body functions, imaging technology to reveal anatomic and functional information continues to flourish in terms of research activity within basic science and engineering fields. For a more in depth review of the role of imaging in systems biology please see [4].

\section{ThE QUEST FOR LESS INVASIVE IMAGING}

Technological advances in the biomedical imaging field have been geared not only towards novel or improved clinical appli- 
cations, but also towards minimal invasive diagnosis and treatment. Biomedical engineers strive to reduce the risk factors associated with each individual modality. Just one example is the effort to decrease the use of gadolinium contrast in magnetic resonance imaging (MRI). Currently under development are noncontrast enhanced angiography techniques, which rely on human physiology and sophisticated acquisition protocols instead of relying on high doses of gadolinium typically associated with magnetic resonance angiography (MRA). On the other hand, it is not a rare case for a traditional application of one modality to be replaced by a safer version of another. Positron emission tomography (PET) imaging of brain physiology, for instance, is giving way to a range of recent MRI techniques. For example, arterial spin labeling (ASL), which is an MRI procedure that demonstrates alterations in brain perfusion, has the potential to be implemented as a routine clinical protocol for diagnosing brain disorders [5].

\section{ULTRASOUND IMAGING}

Real-time three- and four-dimensional (RT3-D, 4-D) technology has been rapidly developing into volumetric ultrasound (US) imaging systems that provide physicians with new dynamic quantitative information about whole organ (cardiac) functionality. Researchers in [6] presented a volumetric segmentation technique for left ventricle (LV) using multiresolution, correlation-based optical flow and active appearance model (AAM) frameworks, respectively. In addition to the volumetric scanning, freehand US image segmentation and registration have been also subjects of study for many researchers as an alternative approach for volume quantification.

Recent advances in the design and fabrication of medical ultrasound transducers have given impetus to develop new probes, multifunctional catheter, and exploratory applications in imageguided interventions [7]. US continues to be used in minimal invasive procedures such as intravascular US (IVUS) due to its real-time, reliable, safe and inexpensive attributes. Consequently, several advanced techniques have emerged in areas of IVUS elastography (IVE), tissue characterization, image fusion, registration and segmentation [8]-[10]. It is worth mentioning that US is being deployed as an effective imaging technology in multimodal acquisition systems for screening and studying biological interactions drug delivery.

\section{MAGNetic Resonance IMAGing AND ITS ApPlicAtion}

The ability to noninvasively image a vast array of soft tissues and internal organs has long established magnetic resonance imaging as one of the most widely utilized imaging modalities. While current research spans a large range of topics, most aspire to render body structure as well as to achieve images which carry increasingly more quantitative information about organ function, physiological performance and disease . For example, in the past few years MRI has entered new domains previously reserved for computed tomography (CT) and PET. MR guided interventions of the breast are also being explored. Cartilage imaging and quantification of its mechanical properties is another active MR application in the treatment of bone disease and injury [11], [12]. Techniques such as perfusion weighted imaging (PWI), which is sensitive to perfusion changes in tissues rich in blood vessels, and diffusion weighted imaging (DWI), which detects the direction of water movement, are finding new diagnostic applications in brain imaging as well as treatment therapy in for stroke. In addition, researchers are actively probing the possibility to utilize these techniques for liver, prostate and pancreatic cancer detection [13].

Whereas traditional MRI has focused on imaging the protons in the body, advances in magnet design have permitted the imaging of a variety of different nuclei. This was previously not feasible due to the weak and/or fast decaying signal of these nuclei. The sodium nucleus, in particular, holds great promise for clinical applications. Changes in sodium tissue concentration (TSC) have been linked to several pathologies including: brain and skin tumors ischemia, osteoarthritic change and are a good indicator of cellular proliferation [14]. Several factors make imaging the sodium nucleus particularly challenging. Sodium has gyromagnetic ratio that is approximately $1 / 4$ that of hydrogen and an abundance in the body that is almost $1 / 1000$ of hydrogen's. The combination of these two factors results in a signal that is roughly 22000 smaller. Additionally, since sodium has a spin of $3 / 2$ as compared with hydrogen's $1 / 2$, its transverse relaxation constant (T2) is significantly shorter and exhibits a fast biexponential decay ranging from $0.5 \mathrm{~ms}$ to 3 ms. Not surprisingly, sodium images are typically characterized by low signal-to-noise-ratio (SNR) and require multiple averages to obtain clinically useful information. There is therefore an inherent tradeoff between image quality and the cost of increasing scan time.

In order to avoid this tradeoff, a two pronged approach can be seen in the literature. Some groups have focused on developing faster acquisition protocols and sampling schemes, a popular choice being hybrid radial sampling. Other groups have focused on the reconstruction aspect of the imaging problem, attempting to improve the obtained image using as few samples as possible. Recent work in this area is encouraging and is likely to yield exciting developments in the coming years.

\section{LONGitudinAl-MULTiMODAL MODELING AND DATA MINING}

Longitudinal multimodal imaging can often enhance medical decisions by fusing together different information sources of pathological processes over time. Current research is devoted to the combination of medical imaging techniques to provide complementary information about the pathology of interest. Innovations include hybrid nanoparticles that act as multiple contrast agents as well as hybrid scanning configurations. In parallel, researchers are developing technology that is able to fuse, compare and evaluate longitudinal imaging studies from different time points through segmentation, registration and interactive visualization techniques [15]-[17]. State of the art methods combine information from spatial anatomical structures and temporal dynamics as well as prior models to aid the modeling process in question. Furthermore, the computation of the modeling schemes is being executed on the graphics processing unit (GPU) to achieve real-time performance for large-scale and interactive processing. Technical advances in multimodal imaging provide us with longitudinal multimodal data sources that are often unexplored in their richness of 
information during the diagnostic decision process. To address this problem researchers from various fields develop learning schemes and intelligent frameworks using statistical models and data mining schemes to provide assisted summarization, fusion and inference of diagnostic information sources. Patient specific and disease specific patient databases start to emerge offering access and management to diagnostic information to aid and improve patient care. [18]-[21]

\section{COMPuter Aided Diagnosis, PACS, AND IMAGE DATABASES-The NeEd FOR SPECIFICITY}

Computer-aided diagnosis (CAD) provides a second opinion to the radiologist [22]-[25] and often finds application in the identification of suspicious abnormal lesions. Recent work is devoted to models for the detection and characterization of potential abnormalities in lung cancer, chest, breast, colon and brain. Commercial systems are starting to translate into clinical practice as well as academic developments. The picture archiving and communication system (PACS) is not only used for image archiving, but also to provide automatic image processing, integrated enterprise workflow and the ability to seamlessly share images among individuals and across reading centers. Furthermore, PACS also provides the ability to digitally enhance images, supporting more detailed and sharper images allowing radiologists to improve their diagnostic capabilities. The integration of CAD and PACS will take on an important role in the routine clinical workflow environment. Future trends involve the integration between PACS and CAD through increased automation and integration of voice control for minimal expert intervention. Large image data as outlined in the previous section, heterogeneous data domains and constantly changing knowledge domains are challenges besides the need for high sensitivity and specificity of CAD algorithms.

\section{SUMMARY}

We have reviewed some of the more recent advances and trends in the area of biomedical imaging but it is certainly not complete. The accelerating pace of technological advances in the field of biological and medical imaging remains high, revealing more information about organs as well as function, place's more demands on the performance of existing algorithms and new systems. Real-time multimodality imaging and image-guided interventions are the other fast growing areas of interdisciplinary research and development. Despite these wonderful advances, mainstream topics such as segmentation, registration and spatial-temporal integration still remain open and require will breakthroughs to improve reliability and automation.

Microelectromechanical systems (MEMS) employed by the medical device industry, appear to be destined for a flourishing biomedical imaging future. The amount of available information coming from heterogeneous data sources to describe an individual patient continues to increase and will be a major concern in workflow in clinical practice. In the future, help may come from CAD systems that integrate PACS to facilitate access to images, medical reports, and laboratory values. Coupled with intelligent data mining schemes this new era of diagnostic computing has the potential to improve diagnostic imaging and patient care. New challenges remain to build computer-aided systems that perform with high sensitivity and specificity in large-scale heterogeneous multimodal data domains.

\section{ACKNOWLEDGMENT}

This article would not have been possible without the outstanding contributions and efforts of N. Lee, A. Katouzin, A. Lorsakul, O. Cohen, and I. Atanasova.

\section{REFERENCES}

[1] H. Kitano, "Systems biology: A brief overview," Science, vol. 295, no. 5560, pp. 1662-1664, 2002.

[2] D. Burstein, "MRI for development of disease-modifying osteoarthritis drugs," NMR Biomed., vol. 19, pp. 669-680, 2006.

[3] A. Yun, Y. Lee, and A. N. Gerber, "Integrating systems biology and medical imaging: Understanding disease distribution in the lung model," AJR, vol. 186, pp. 925-930, 2006.

[4] K. Armen, T. Song, Q. Duan, M. Neimark, M. Po, J. Gohagan, and A. Laine, "A review of imaging techniques for systems biology," $B M C$ Syst. Biol., vol. 12, p. 74, Aug. 2008.

[5] I. Asllani, "Multivariate and univariate analysis of continuous arterial spin labeling perfusion MRI in Alzheimer's disease," J. Cereb. Blood Flow Metab., vol. 28, pp. 725-736, 2008.

[6] E. D. Angelini, A. F. Laine, S. Takuma, J. W. Holmes, and S. Homma, "LV volume quantification via spatiotemporal analysis of real-time 3-D echocardiography," IEEE Trans. Med. Imag., vol. 20, no. 6, pp. 457-469, Jun. 2001.

[7] C. A. Linte, A. Wiles, J. Moore, C. Wedlake, and T. M. Peters, "Virtual reality-enhanced ultrasound guidance for atrial ablation: In vitro epicardial study," in MICCAI 2008, Part II, 2008, pp. 644-651.

[8] S. B. W. Wein, A. Khamene, M. R. Callstrom, and N. Navab, "Automatic CT-ultrasound registration for diagnostic imaging and imageguided intervention," Medical Image Analysis, vol. 12, pp. 577-585, 2008.

[9] L. R. Maurice, J. Fromageau, M. R. Cardinal, M. Doyley, E. De Muinck, J. Robb, and G. Cloutier, "Characterization of atherosclerotic plaques and mural thrombi with intravascular ultrasound elastography: A potential method evaluated in an aortic rabbit model and a human coronary artery," IEEE Trans. Inf. Tech. Biomed., vol. 12, pp. 290-298, 2008.

[10] K. A. B. Baseri, E. E. Konofagou, and A. F. Laine, "An alternative approach to spectrum-based atherosclerotic plaque characterization techniques using intravascular ultrasound (IVUS) backscattered signals," in MICCAI 2008, 2008, vol. CVII, pp. 53-62.

[11] A. Werner, Kaiser, Stefan, O. R. P. Pascal, and A. T. Baltzer, "MRIguided interventions of the breast," J. Magn. Resonance Imag., vol. 27, pp. 347-355, 2008.

[12] R. Kijowski, M. Tuite, L. Passov, A. Shimakawa, H. H. Scott, and B. Reeder, "Cartilage imaging at 3.0T with gradient refocused acquisition in the steady-state (GRASS) and IDEAL fat-water separation," $J$. Magn. Resonance Imag., vol. 28, pp. 167-174, 2008.

[13] S. Siraj, Abdullah, J. B. Pialat, M. Wiart, F. Duboeuf, J.-Y. Mabrut, B. Bancel, A. Rode, C. Ducerf, J. Baulieux, and Y. Berthezene, "Characterization of hepatocellular carcinoma and colorectal liver metastasis by means of perfusion MRI," J. Magn. Resonance Imag., vol. 28, pp. 390-395, 2008.

[14] V. Schepkin, T. Chenevert, K. Kuszpit, K. Lee, C. Meyer, T. Johnson, A. Rehemtulla, and B. Ross, "Sodium and proton diffusion MRI as biomarkers for early therapeutic response in subcutaneous tumors," $J$. Magn. Resonance Imag., vol. 24, pp. 273-278, 2006.

[15] W. J. M. Mulder, D. P. Cormode, S. hak, M. E. Lobatto, S. Silvera, and Z. A. Fayad, "Multimodality nanotracers for cardiovascular applications," in Nature Clinical Practice Cardiovascular Med., Mar. 28th, 2008, vol. 5, pp. 103-111.

[16] Q. Duan, E. D. Angelini, S. Homma, and A. F. Laine, "Real-time segmentation of $4 \mathrm{D}$ ultrasound by active geometric functions," in IEEE ISBI 2008, 2008, pp. 233-236.

[17] T. Song, V. S. Lee, H. Rusinek, Q. Chen, L. Bokacheva, and A. Laine, "Segmentation of 4D MR renography images using temporal dynamics in a level set framwork," in 5th IEEE Int. Symp. Biomedical Imaging: From Nano, 2008. 
[18] J. Curtis, H. Cheng, E. Delzell, D. Fram, M. Kilgore, K. Saag, H. Yun, and W. DuMouchel, "Adaption of Bayesian data mining algorithms to longitudinal claims data. Coxib safety as an example," Medical Care, vol. 46, pp. 969-975, Sep. 2008.

[19] J. J. Caban, N. Lee, S. Ebadollahi, A. Laine, R. DeLaPaz, and J. R. Kender, "Concept detection in brain MR images using multi-modal information," presented at the Society of Imaging Informatics in Medicine (SIIM) Conf., Charlotte, NC, Jun. 2009, , to be published.

[20] S. T. Mahmood, F. Wang, D. Beymer, A. Amir, M. Richmond, and S. N. Hashmi, "AALIM: Multimodal mining for cardiac decision support," Computers in Cardiology, vol. 34, pp. 209-212, 2007.

[21] S. M. Brown, S. K. Shah, R. C. Pais, and Y.-Z. Lee, "Database design and implementation for quantitative image analyais research," IEEE Trans. Inf. Technol. Biomed., vol. 9, no. 1, pp. 99-108, Mar. 2005.
[22] K. Doi, "Computer-aided diagnosis in medical imaging: Historical review, current status and future potential," Computerized Medical Imaging and Graphics, vol. 31, pp. 198-211, 2007.

[23] M. L. Giger, "Computer-aided diagnosis in medical imaging - A new era in image interpretation," Medical Imaging ULTRASOUND, pp. 75-78, 2008.

[24] T. Valenza, "PACS: Top trends for today and tomorrow," Medical Imaging, pp. 22-29, May 2008.

[25] R. D. Barlow, "Larger volume data sets redefining PACS workflow demands complicating patchwork integration," Outpatient Care Technology Jun./Jul. 2008 [Online]. Available: http://www.coactiv.com/ pdf/2008/LargerVolumeDataSetsRedefiningPACS.pdf 